\section{ON SPACE TIME FOAM}

MILÁN, ITALIA

TOMÁS SARACENO

2012
El trabajo de Saraceno plantea un cruce entre ciencia, arte y técnica que se pone al servicio de una reflexión social: sus membranas transparentes y habitadas retoman propuestas de la arquitectura radical de los setenta.

PALABRAS CLAVE: INSTALACIÓN - ITALIA, MEMBRANA, PVC, PROXÉMICA, ARTE Y ARQUITECTURA
Saraceno's work raises crossings between science, art and technology and addresses social matters: his

transparent, inhabited membranes seem to follow the thread of 1970's Radical architects.

KEYWORDS: INSTALLATION - ITALY, MEMBRANE, PVC, PROXEMICS, ART AND ARCHITECTURE.

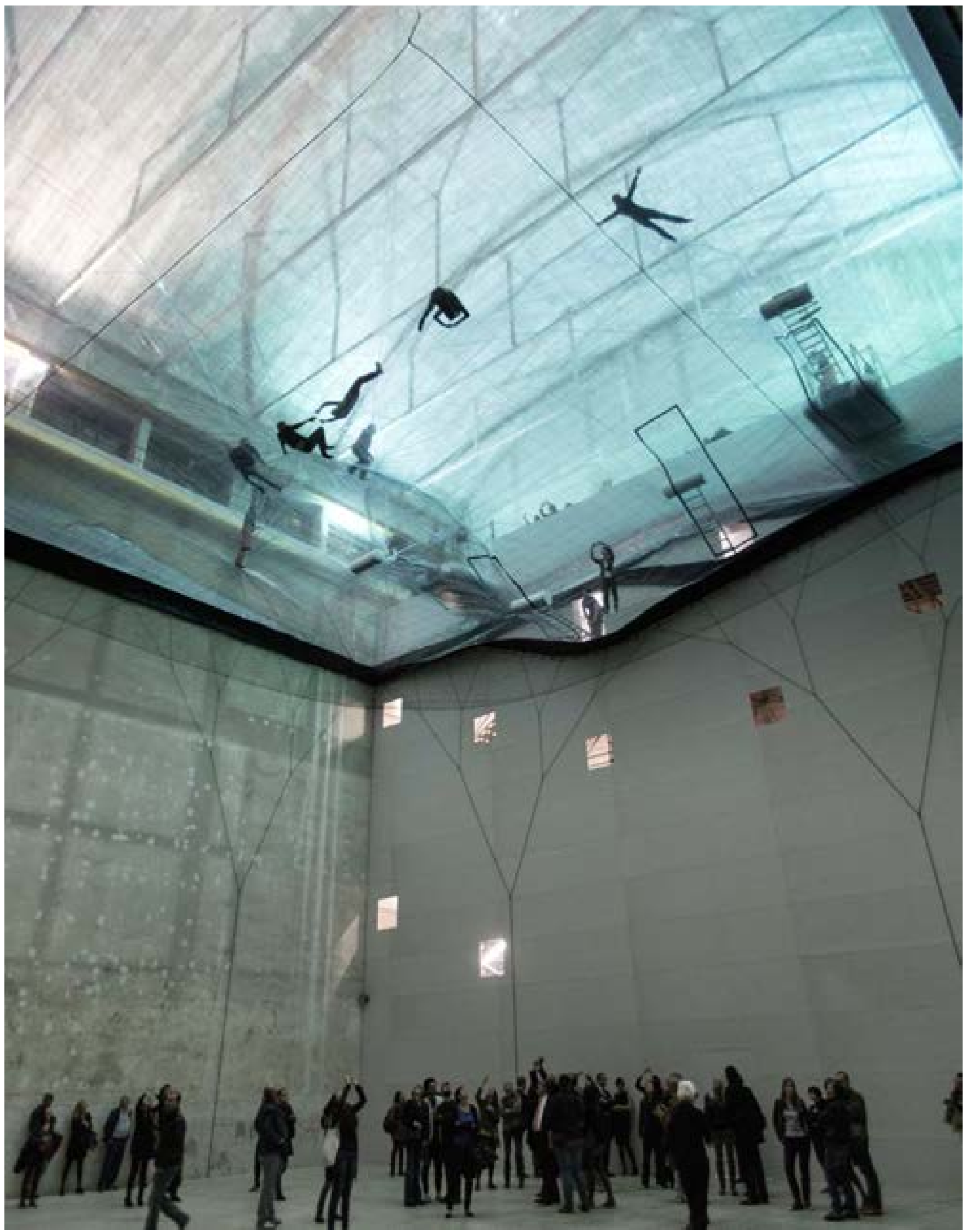

Fotografías de Alessandro Coco, 2012. Fuente: Archivo Tomás Saraceno Studio.

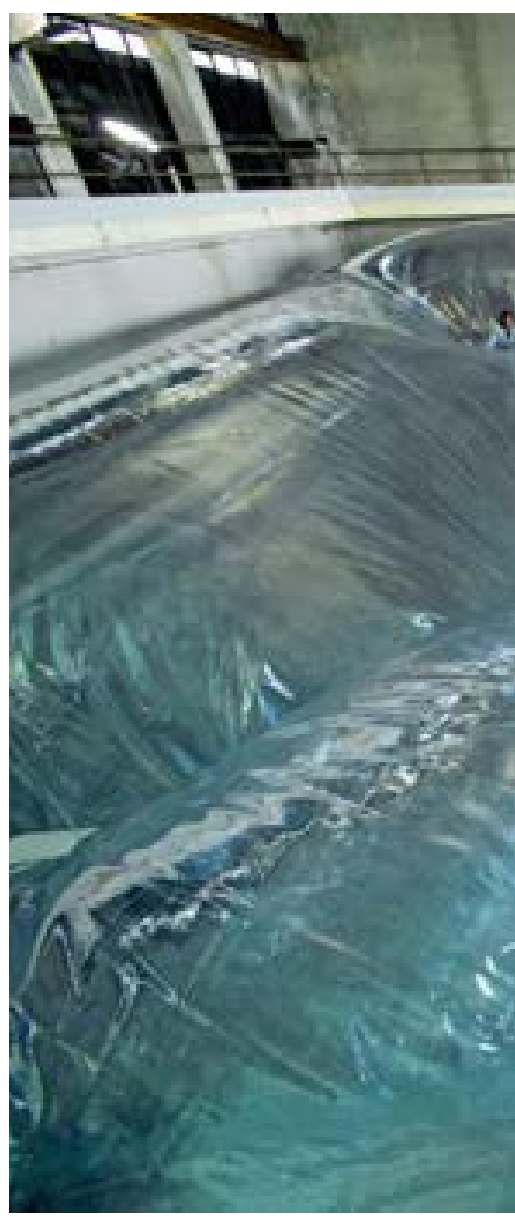




\section{¿DÓNDE ESTÁN TODOS?}

\section{ENTREVISTA DE FILIPA RAMOS}

Iniciar una conversación ${ }^{1}$ con el artista argentino Tomás Saraceno (nacido en Tucumán en 1973) es como subir a una montaña rusa. En cuestión de segundos él puede pasar de los más recientes descubrimientos de la física cuántica a una descripción minuciosa de la mecánica utilizada por las arañas para hacer viajes aéreos. Da la impresión de que es su inmensa curiosidad y entusiasmo lo que le da forma a sus proyectos, enfocados en la ampliación de los límites del cuerpo y de la percepción antes que en la visualidad.

Para On Space Time Foam, su instalación en HangarBicocca en Milán, Saraceno imaginó una enorme membrana transparente (a la que graciosamente llama "la lasaña") donde los visitantes pueden entrar. Plegada en tres capas, la instalación se encuentra suspendida del suelo a una altura equivalente a seis pisos y ofrece una experiencia corporal radical. Ocho breves preguntas se sucedieron en esta conversación para intentar entender las ideas detrás de esta nueva obra y los universos en constante expansión de Tomás Saraceno.

\section{¿QUÉ ES EL ESPACIO?}

Lo que yo me pregunto es qué hay antes del espacio y el tiempo, y cuál es ese momento singular en que comienza la existencia de las cosas: esa espuma cuántica que es un espacio de burbujas y chispas. En YouTube hay videos que muestran lo que pasa cuando pones una mezcla de maicena y agua sobre un parlante (un "fluido no-newtoniano"2). Es muy impresionante, la mezcla va tomando las formas más extrañas con la vibración del sonido -formas similares al dibujo de Paul Davie que usamos para el proyecto en HangarBicocca- a modo de esferas que ebullen y se rompen como membranas.

Pienso en el espacio como un tímpano, una membrana que permite escuchar porque vibra. El universo podría ser una membrana, de alguna manera cercana a lo que tenemos en esta exhibición: múltiples capas de membranas vibrando como universos unidimensionales y planos. $\mathrm{Si}$ una capa toca a otra, hacer colapsar espacio y tiempo formando un hoyo negro. La teoría de cuerdas 3 , o más bien algunos de sus estudiosos, han especulado que el Big Bang -el origen del cosmos conocido- surgió a partir de la colisión de dos enormes membranas, la que provocó la expansión del espacio y la materia.

\section{¿QUÉ ES EL PESO?}

En un contexto astrofísico llamaría al peso masa, que es lo que envuelve y da forma al espacio y al tiempo. La masa es siempre relativa. En un vuelo parabólico, el avión se mantiene en caída libre y uno no pesa nada. Según Einstein, de la misma manera en que una esfera de cierta magnitud sobre una tela elástica estira el tejido y lo afloja, del mismo modo los planetas y las estrellas tensionan el espacio y el tiempo. Una bolita moviéndose sobre la tela será atraída hacia la esfera mayor, como pasa con la Tierra y el Sol, pero no chocará con ella mientras se mantenga en movimiento.

Eso es lo que pasaba en esta instalación: si una persona muy grande entra, allí la membrana se hunde. Si cinco personas entran y se acercan demasiado, para ellos será muy difícil salir de allí, porque su propio peso los empujará hacia abajo: se produce una especie de agujero negro social. El proyecto es en definitiva un intento por vincular orden cósmico y orden social, apoyado en el concepto de proxémica que elaboró el antropólogo Edward T. Hall.

\footnotetext{
Esta entrevista, realizada a Tomás Saraceno por la curadora portuguesa Filipa Ramos, fue publicada en el sitio web de la revista DOMUs en octubre de 2012. La versión original en inglés puede revisarse en

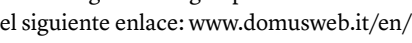
interviews $/ 2012 / 10 / 29 /$ where-is-everybody-.html. Agradecemos a Carmen Figini de DOMUs y a Filipa Ramos por su gentil autorización a publicar este texto.
}

Un fluido newtoniano es una sustancia homogénea de viscosidad constante, por lo que se deforma de manera continua en el tiempo ante la aplicación de una solicitación, cualquiera sea hace informe; siempre adopta la forma de su contenedor, como el agua. En cambio, la viscosidad (resistencia a fluir) de un fluido no newtoniano varía con el gradiente de tensión aplicada: estos fluidos se deforman en la dirección de esa fuerza. su magnitud. Su poca cohesión intermolecular la

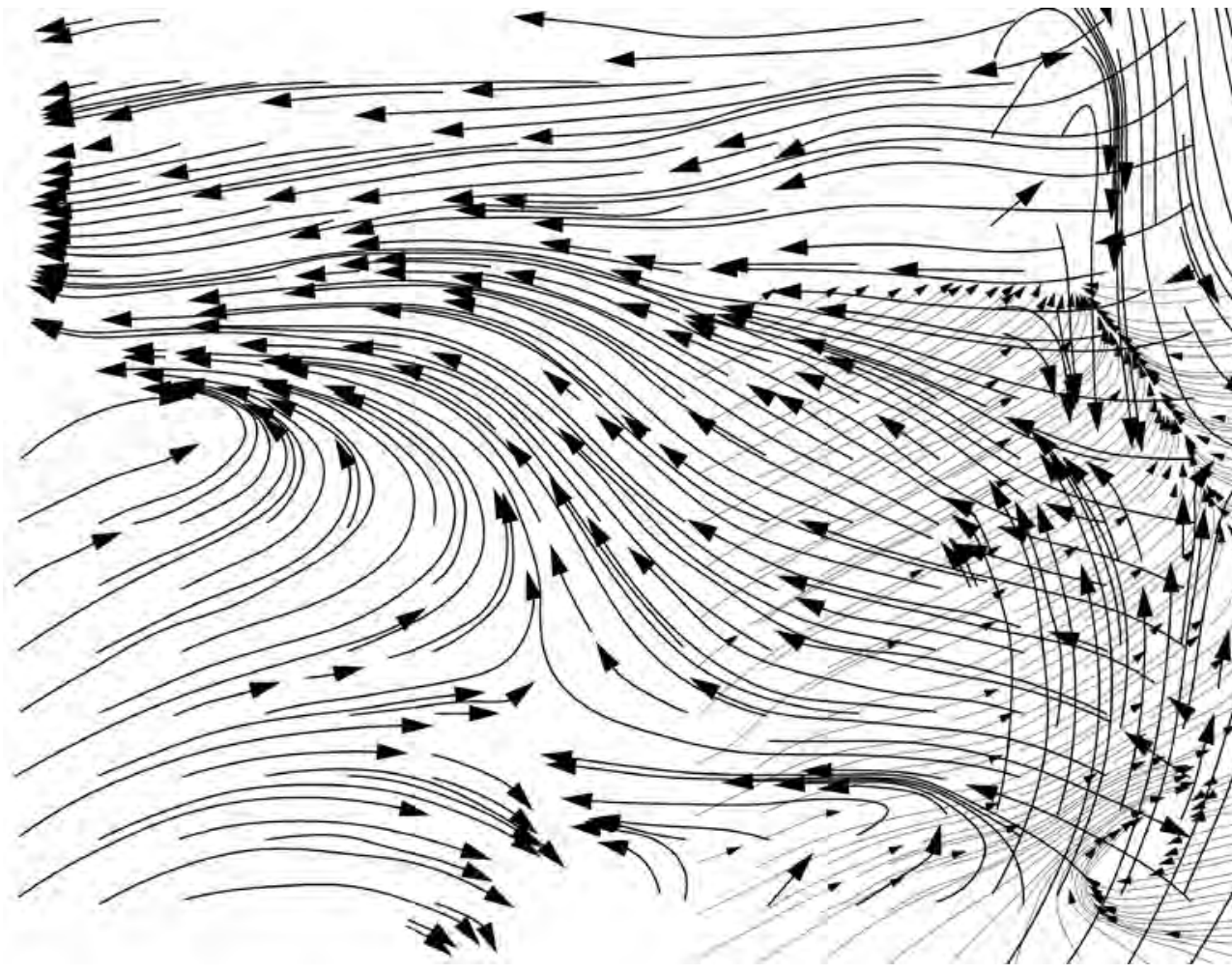

Collage del autor, 2012. Fuente: Archivo Tomás Saraceno Studio.

\section{¿QUÉ ES LA GRAVEDAD?}

Continuando con la proxémica: imagina una habitación repleta de personas. De repente, una de ellas recibe la noticia de un reconocimiento muy importante. A partir de ese momento, una conversación con esa persona implicará un aumento en la distancia entre los interlocutores: se formará un espacio entre ambos. La proxémica se ocupa de eso: analiza la influencia del conocimiento y la cultura en los modos en que el espacio se condiciona y se mide. Este proyecto comprende una demostración física de cómo los individuos forman espacio al relacionarse unos con otros: se trata de una estructura similar a la de una lasaña, sin espacio entre sus hojas. Al entrar, el propio peso del cuerpo forma espacio, estirando y abriéndose paso entre el material de la membrana, a medida que se mueve. iEs difícil imaginar un espacio más correlacionado y codependiente que ese! Estando allá arriba, cualquiera de mis movimientos condicionará los tuyos y los de los demás.

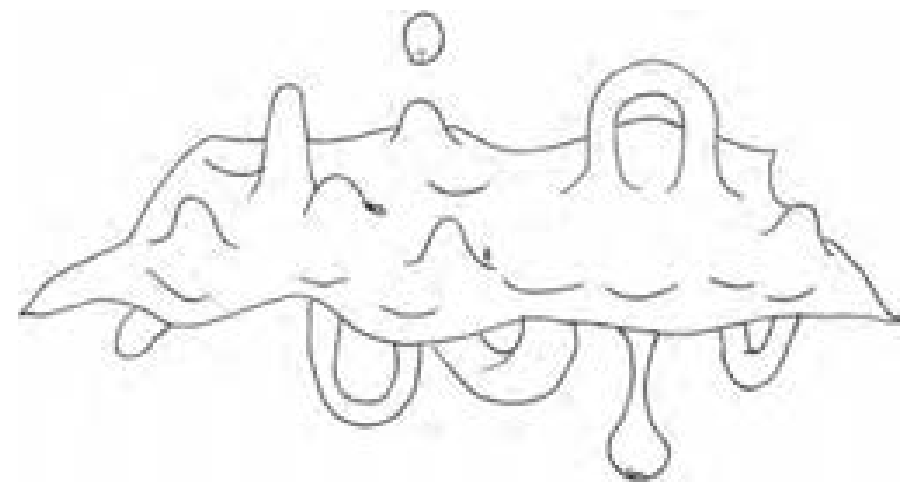

La teoría de cuerdas apareció como una 3 de las respuestas para la formulación de una teoría cuántica de la gravedad. Pretende, además, ser una descripción completa, unificada y consistente de la estructura fundamental del universo. La idea esencial detrás de ella es que todas las diversas partículas "fundamentales" del modelo estándar son en realidad manifestaciones variadas de un objeto básico: una cuerda. 


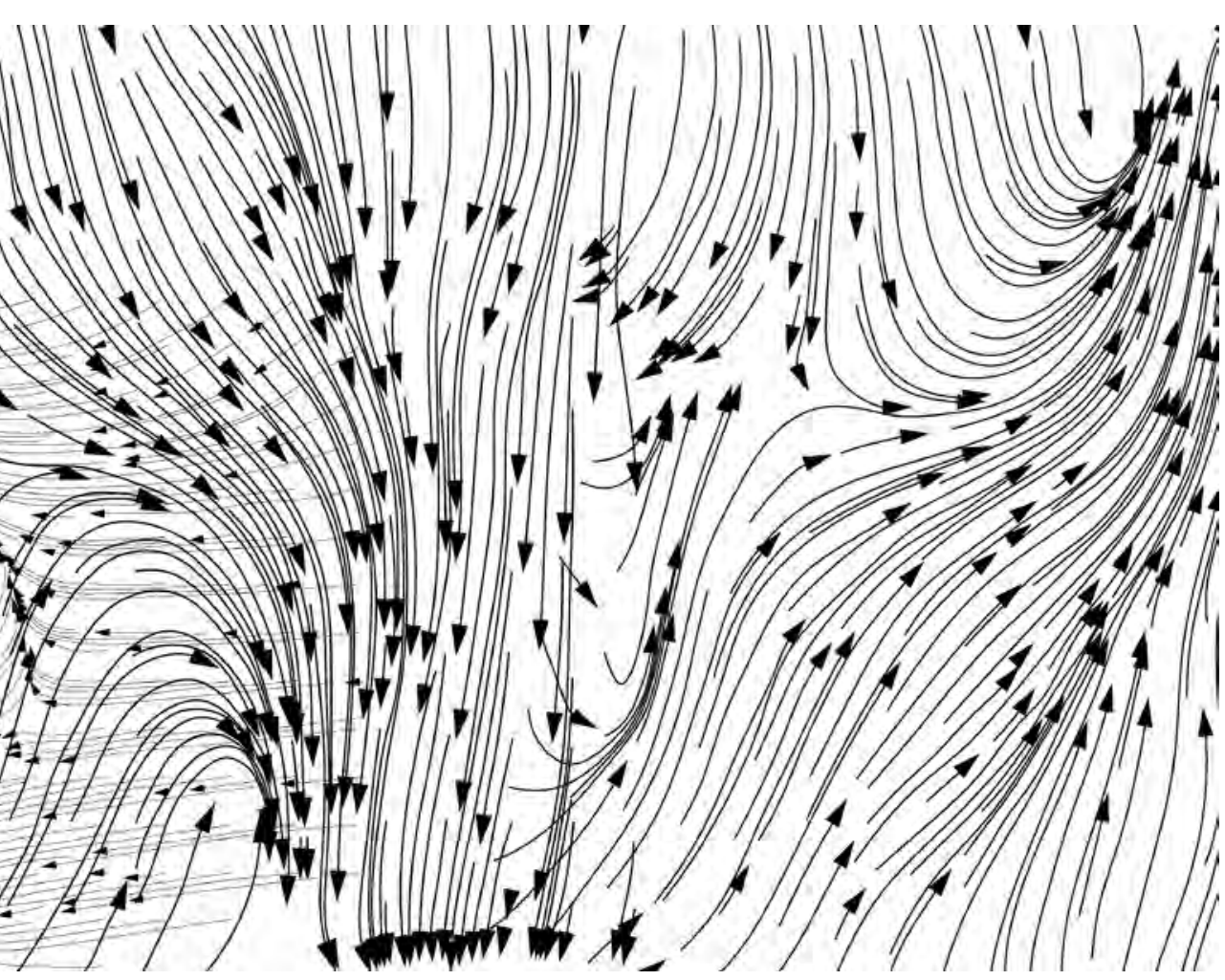

¿QUÉ ES EL RITMO?

Es cuando algo suena bien, incluso cuando no sabes qué es. Sucede en la poesía, cuando lees algo y tiene una buena cadencia. Se trata de alternancia y repetición, marcadas por la sucesión regulada de elementos opuestos, la dinámica de los latidos fuertes y débiles, el sonido activo y la pausa inaudible pero intencionada, la nota larga y la corta.

La paradoja de Fermi ilustra la contradicción entre la alta probabilidad de existencia de vida en el espacio exterior y la completa ausencia de contacto con ella. Enrico Fermi se hizo famoso al exclamar: "¡¿Dónde están todos?!” preguntándose por el paradero de otros seres en el universo. Probablemente, nunca se ha logrado tener contacto con nadie más en el universo porque como humanidad nunca hemos logrado tocar al unísono un solo acorde. He estado hablando con Bruno Latour sobre la colaboración en un proyecto para una obra de teatro que está haciendo en Toulon. Pensé en transformar todas las redes y las esferas de la pieza de la Bienal de Venecia, Galaxy forming along filaments, like droplets along strands of a spider's web, en cuerdas, en un instrumento enorme y coral listo para ser tocado.

Se trata de inventar un instrumento que todos los seres humanos en el planeta puedan tocar al mismo tiempo, de modo que cuando uno toque una cuerda, esta tenga una repercusión en todas las otras. Eso nos sintonizaría a todos. Cuando seamos capaces de producir armonía como una sola especie, tal vez alguien allá afuera nos escuche. Eso es lo que ocurría en el HangarBicocca en Milán, era un intento por hacer que cada individuo se involucrara y sintonizara con los demás.
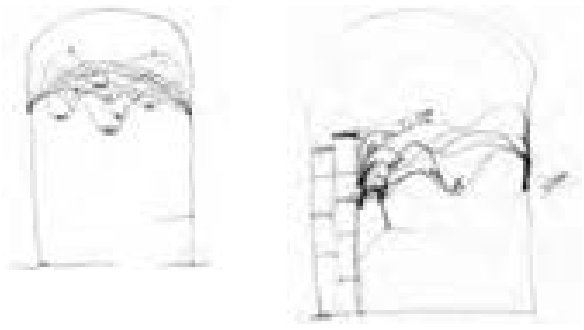

Bosquejos del autor, 2012. Fuente: Archivo Tomás Saraceno Studio.
¿QUÉ ES EL DESPLAZAMIENTO?

Stephen Hawking cuenta una hermosa historia para describir el concepto de desplazamiento. Él recuerda cómo "hace unos años el ayuntamiento de la ciudad italiana de Monza privó a la gente de tener peces en peceras redondas, explicando que es cruel mantener a un pez en una pecera pues los lados curvos dan a los peces una visión distorsionada de la realidad". Además de la importancia que dicha medida podría tener para los peces cautivos, la historia plantea una cuestión filosófica interesante: ¿Cómo sabemos que la realidad que percibimos es "real"? El pez ve una versión de la realidad que es diferente a la nuestra, pero ¿cómo podemos estar seguros de que es menos real? Por lo que sabemos, nosotros también podríamos estar pasando nuestras vidas enteras viendo el mundo a través de un lente deformador.

El desplazamiento es como ser pez dentro de una pecera.

\section{¿QUÉ ES LA ARQUITECTURA?}

¿Sabías que la mayoría de las arañas son ciegas? Ellas perciben el mundo a través de vibraciones. También hacen algo que se conoce como "viaje en globo": icuelgan su fibra de seda de un árbol y cuando el viento es lo suficientemente fuerte, despegan! Pueden viajar largas distancias y cruzar continentes enteros. ¡Debe ser increíble ser ciego y volar sobre el mar, como en un globo!.

Me gusta pensar en la arquitectura como un sistema informático, o como la composición de un poema. La arquitectura no sólo como la construcción de edificios, sino también como una forma de organizar y entender algo que no está exclusivamente relacionado a los seres humanos.

\section{¿QUÉ ES UNA UTOPÍA?}

Es una necesidad constante. La utopía también puede estar en otra dimensión, como los sueños, y de la misma forma es una necesidad, porque todo el mundo necesita dormir y soñar: pasamos un tercio de nuestra vida en otra dimensión, soñando. A veces te despiertas con pesadillas horribles, pero en otras ocasiones tienes un sueño hermoso que desearías continuar. Cuando se está despierto, lo importante es reunir la persistencia y resistencia necesaria para que el sueño pueda ocurrir, o continuar.

\section{¿QUÉ ES EL DESEO?}

Hace un tiempo la madre del paracaidista Felix Baumgartner publicó un dibujo hecho por él cuando tenía cinco años, en el que se representaba a sí mismo saltando en paracaídas. ¡Tenía un deseo enorme y se las arregló para cumplirlo! Eso muestra cómo uno puede comprometerse con sus sueños y cumplirlos, incluso de forma inconsciente.

Creo que sería fantástico si, después de mostrar esta pieza en el HangarBicocca, ella pudiera convertirse en una biósfera flotante sobre las Maldivas. Tuve la fortuna de ser el primer artista visitante en el Center for Art, Science \& Technology (CAST) en MIT; tal vez junto a ellos y PIRELLI podamos hacer que las ciudades que vuelan despeguen, una vez más. ARQ

ON SPACE TIME FOAM / Arquitecto: Tomás Saraceno / Colaboradores: Lindstrand Technologies y European Space Agency (ESA) / Curador: Andrea Lissoni / Ubicación: HangarBiccoca, Via Chiese 2, Milán, Italia / Promotor: PIRELLI / Sistema constructivo: membrana plástica transparente, tensores de acero / Presupuesto: sin datos / Superficie construida: $1,200 \mathrm{~m}^{2}$ en tres estratos de $400 \mathrm{~m}^{2} /$ Año de construcción: 2012 / Fotografías: Alessandro Coco, Tomás Saraceno Studio. 


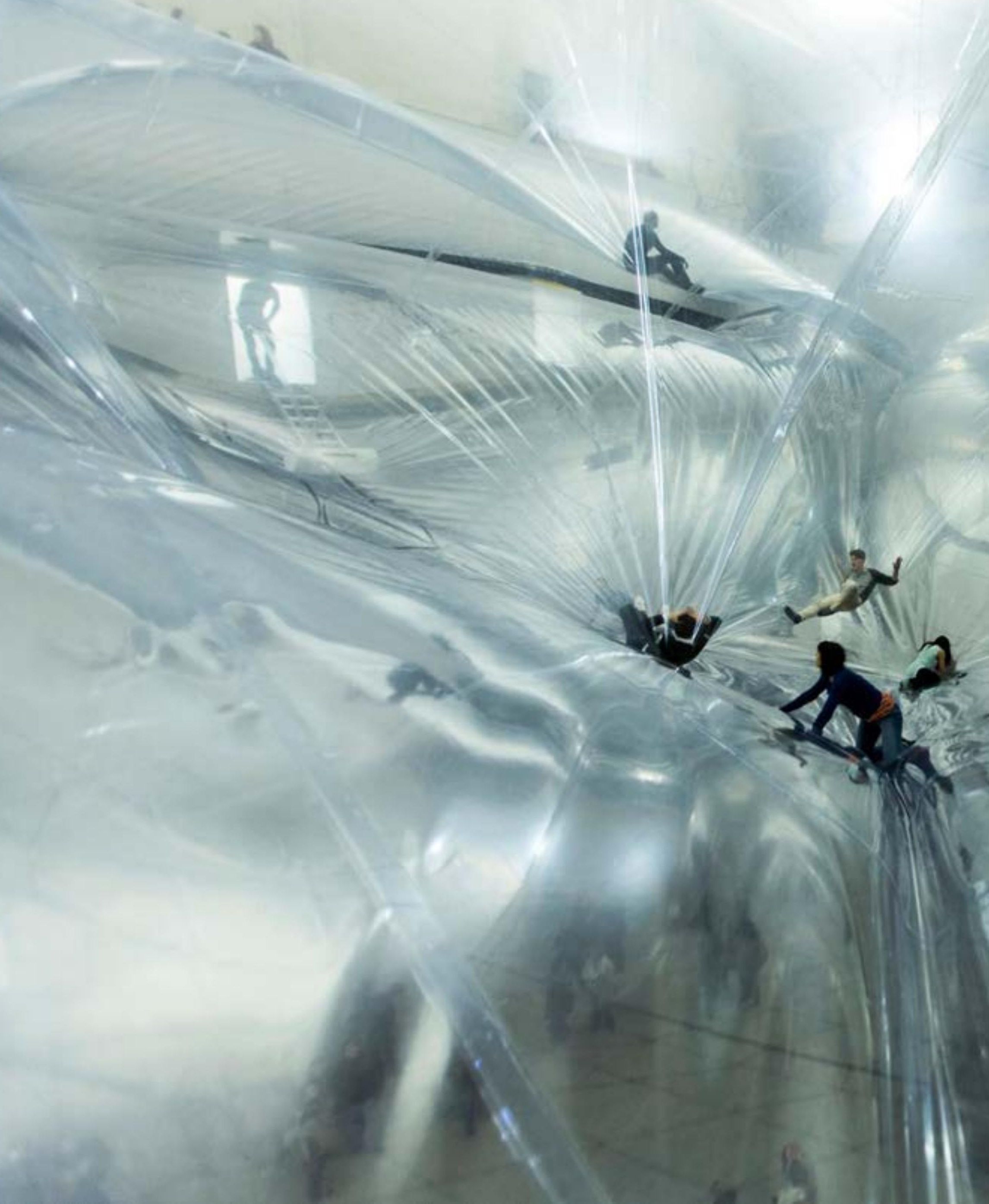



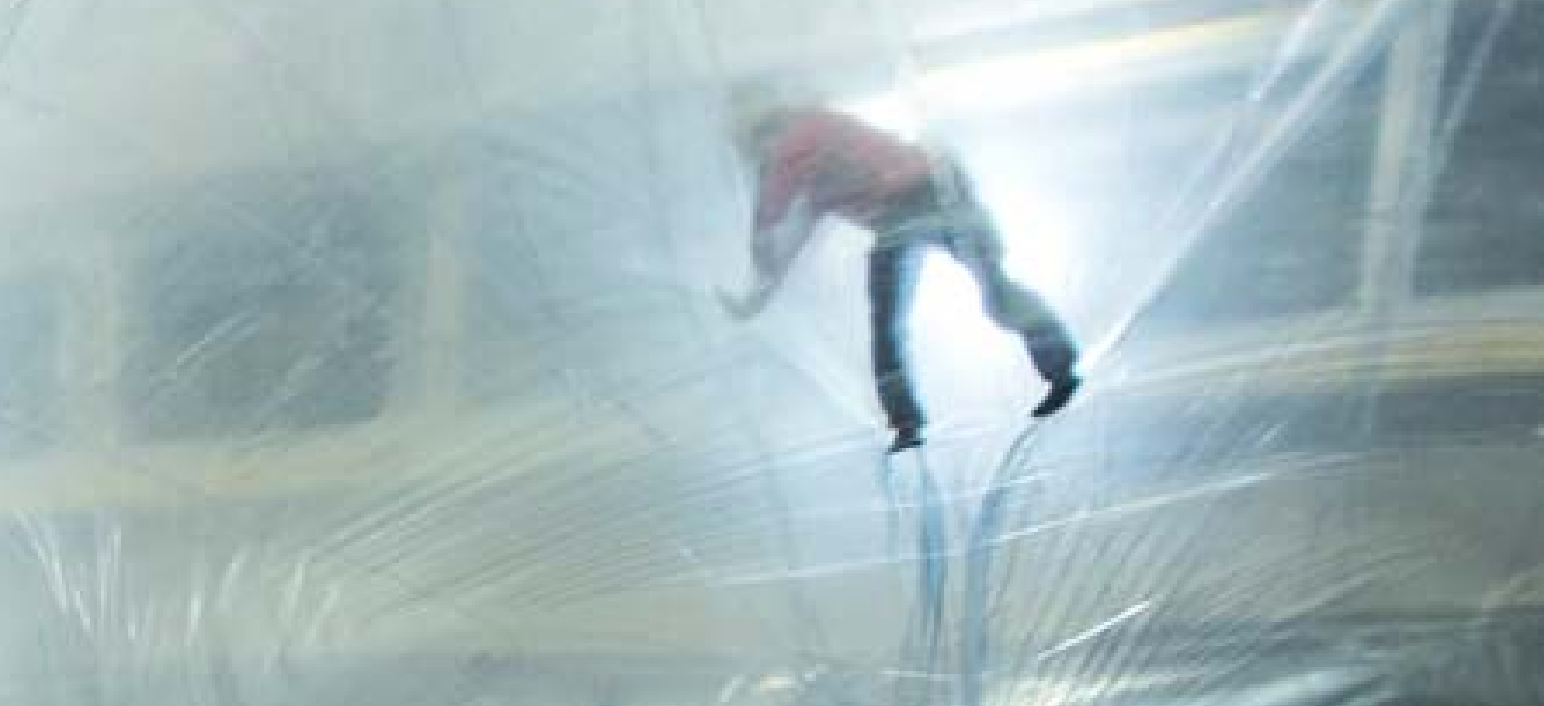

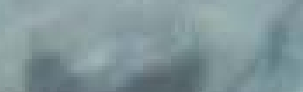
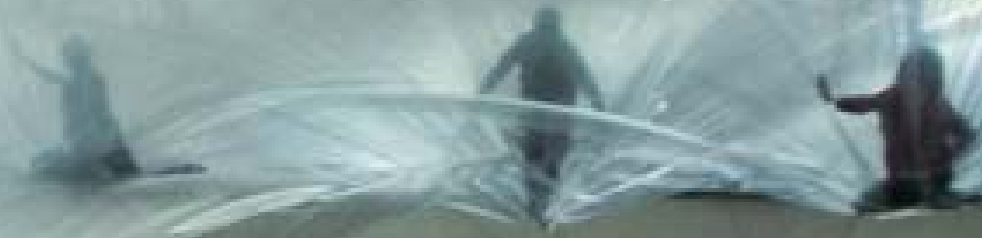

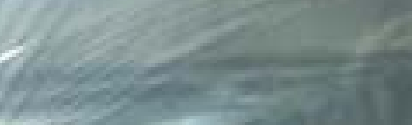

